\title{
Research on Collaborative Transmission Technology of Wireless Sensor Network Based on Energy Saving
}

\author{
TIAN Ye-fei \\ ChongQing college of electronic engineering, Chongqing, China \\ Email:183348163@qq.com
}

Keywords: Collaborative Transmission Technology, Wireless Sensor Network, Energy Saving

\begin{abstract}
In this paper, we study the method of wireless sensor network based on energy-saving wireless sensor network, and put forward the comparison criteria of wireless transmission network single transmission system and performance of collaborative transmission system based on space-time block code. Aiming at the characteristics of wireless sensor network energy limitation, and the threshold level of the optimal transmit power of the cooperative transmission system is calculated. The simulation results show that the threshold level can minimize the total transmission power of the wireless sensor network under the condition of satisfying the certain probability of the system.
\end{abstract}

\section{Introduction}

Wireless sensor network is a large number of sensor nodes to wireless communication technology as a support, through a certain topology of the network, is one of the current hot spots in the military, commercial, medical, environmental, intelligent transportation and other aspects of important applications. It is characterized by the limited energy, large scale, self - organization, and the collection of massive information for fusion, which is transmitted by the sensor nodes through the wireless environment. In the wireless sensor network, energy efficiency is a decisive factor. It is particularly important because wireless transmission usually consumes most of the energy of the sensor battery. The transmission energy efficiency can be enhanced by the diversity of the antenna, while the space-time block codes (STBCs) are a very effective spatial diversity Technology, which has the characteristics of low complexity and high bandwidth utilization, so it is very suitable for wireless sensor networks, and has important application value. Synchronous STBCs not only improve the energy efficiency of transmission, but also disperse the energy consumption between multiple nodes and it is conducive to extending the network survival time.

The energy saving method of wireless sensor network based on STBCs is carried out under good synchronization condition. It is studied in the case of energy limited how to control how many nodes cooperate to save power, but did not propose effective power control Algorithm, we do not quantify the cooperative and non-cooperative states. We propose a comparison criterion of STBCs wireless transmission network single transmission system and cooperative transmission system performance and calculate the optimal transmission power threshold level, which provides reference for energy-saving design of wireless sensor networks.

\section{The Related Research on Wireless Sensor Network}

Wireless Sensor Networks (WSN) is a hot research area in the world that has attracted worldwide attention, highly interdisciplinary and highly integrated knowledge. Conceptually, it consists of a large number of micro-sensor nodes, which are randomly deployed in the monitoring area, and form a single-hop or multi-hop self-organizing and adaptive network system through wireless communication to sense, collect and process the network covers the information of the monitored object in the area and sends it to the observer. At present, the wireless sensor network has been widely used in military defense, industrial and agricultural control, urban management, biomedical, environmental testing, emergency relief, remote control of dangerous areas and other fields. With the rapid development of wireless communication technology, Internet of Things technology, its future application prospects are very broad. 
As with other communication networks, wireless sensor networks have common requirements in terms of channel capacity, reliability, and delay design. In addition, due to some special characteristics of the sensor nodes, such as intensive delivery, long working cycle, non-contact environment, the battery is not rechargeable and its energy requirements are very harsh. The effectiveness of energy utilization becomes an important indicator that must be given priority in the design of wireless sensor networks. There are three main ways to realize the energy-saving goal. First, it reduces the energy consumption of the circuit when the single sensor node is working. The second is to reduce the transmission energy consumption during the communication process. Thirdly, the hibernation-activation mechanism is introduced to optimize the global energy consumption. The energy consumption of the circuit belongs to the category of sensor hardware design. The sleep mechanism has also been widely used in the sensor network, and the transmission energy consumption has become the sensor network due to the many advanced technologies such as self-organizing network, adaptive routing and wireless communication channel the focus of energy conservation research.

As one of the key technologies of 4G mobile communication smart antenna, MIMO (Multiple-Input Multiple-Output) system has been proved to greatly enhance the channel capacity in fading channel. Compared to SISO (Single-Input Single-Output), MIMO can maintain lower energy cost and bit error rate while providing the same bit rate. This provides a research idea for introducing MIMO technology into wireless sensor networks to reduce transmission energy consumption. However, by limiting the physical size of the sensor nodes, it is not feasible to design a multi-antenna directly on a node. This requires the concept of a cooperative MIMO (MIMO) or virtual MIMO (MIMO), that is, through a combination of single antenna nodes to form a de facto distributed MIMO transmission system. The advantages of MIMO transmission in diversity, multiplexing, and beam forming are then applied to wireless sensor networks.

The research on cooperative MIMO communication has been very intensive in recent years. In a cluster-based cooperative MIMO communication protocol is proposed. Given the limitation of the energy consumption of the sensor nodes, the formulas of the flattened Rayleigh channel are given. In an optimal allocation strategy of power and time slot under single hop cooperative MIMO communication is proposed, which is equivalent to the total energy consumption of the system. This assumption is feasible in long-distance communication, but in short-range communications, each node's circuit power consumption and transmission energy consumption is comparable or even sometimes dominant, and therefore cannot be ignored. By comparing the total energy consumption at various fixed-error rates (Co-MIMO, Co-MISO, Co-SIMO), the researchers found that there was no single most for the wireless sensor network excellent transmission mode. Depending on the conditions, the optimized energy efficiency model will be converted between SISO and Co-MIMO / MISO, single-hop and multi-hop. All of the proposed mode conversion strategies are based on the transmission distance information, but the distance information in the sensor network is not explained. In fact, for distributed sensor nodes, the more practical and easy to measure mode conversion parameters are path loss (path-loss).

\section{Co - MIMO Communication Model Based on Cluster}

For ease of analysis, this paper assumes that there is no difference between all sensor nodes. These nodes form a route through a distributed networking algorithm (such as LEACH). In each cluster, nodes with the highest SNR are selected as cluster-head, and cooperative node angular position information is not considered.

\section{Resource Allocation Optimization Algorithm}

There are two parameters that affect the allocation of system resources, time slot and power. By adjusting the intra-group broadcast and inter-group transmission of the time slot and the average power, Co-MIMO system can be in the code rate and energy consumption can be optimized between the balances. Since the ITA time slot CSI is known, it is assumed that the intra-group 
broadcast SIMO channel is the Rayleigh fading for all states. Since the ITE time slot CSI is unknown, the non-states are infiltrated by the Rayleigh fading for the Co-MIMO channel. All channels are flat channels in the frequency domain.

Through the energy allocation, cluster-based cooperative communication is superior to SISO mode in energy consumption and reliability communication. More collaborative nodes mean smaller interrupt probability. However, taking into account the time division system in addition to Alamouti STBC coding coding rate $\mathrm{R}$ are less than 1, this improvement in the probability of interruption can be seen as a loss of bit rate in exchange for. And Alamouti coding because the coding rate of 1 , you can maximize the probability of interrupt and code transmission rate to find a balance between.

\section{Sleep Energy Saving Strategy Analysis}

Sleep energy saving strategies are divided into two categories: asynchronous clock sleep and synchronous clock sleep. Asynchronous Clock Sleep Mechanism: Each node in the system runs a separate clock and performs a separate sleep schedule. The detailed communication mechanism shown in Figure 2, the coordinator will command or data sent to the designated terminal device, the parent node, that is, the router. After the terminal node wakes up, first send a data request Request to its parent node to see if there is any data sent to itself. If the parent node has the data sent to the child node, it will send the data immediately after receiving the data request of the child node. If the parent node does not save the data, it will reply to Ack without data. The terminal node goes to sleep again after a communication is completed, or after receiving the Ack of the parent node. The advantage of the asynchronous clock hibernation mechanism is that all nodes in the wireless network maintain independent clocks without the use of complex clock synchronization algorithms that are easy to implement. Disadvantages are: (1) because the clock is not synchronized, regardless of the parent node with or without data, the terminal node to wake up every time to send the query command, resulting in invalid communication overhead. (2) because the parent node (that is, the routing node) to wake up the terminal node to save data. But the node's storage resources are limited, when the child nodes and traffic is more likely to cause data overflow. (3) asynchronous clock communication mechanism in the routing node cannot sleep, in the complex large-scale network, the greater the greater the energy consumption of the routing node. (4) sensor cannot achieve active upload data, each communication must first request data from the terminal node, and then wait for data, resulting in unnecessary communication costs.

Synchronous clock sleep mechanism: all nodes in the system clock synchronization, you can achieve the same sleep, while wake up after the data can be directly communicated. The main advantages: (1) after each wake-up, no need to send data requests to the parent node; (2) router can achieve sleep; Disadvantages: the traditional clock synchronization method to achieve more difficult to increase the communication overhead, the accuracy of the larger network size high. Different clock synchronization algorithms, energy consumption are not the same.

With the increasing cost of wireless communication, WIFI coverage is more and more widely, because WIFI (802.11) and ZigBee (802.15) are using ISM band, the study found that 802.15.4 RF module can receive to the periodic Beacon signal in the 802.11 network. Using this feature, some scholars have studied the ZigBee-WiFi network based on energy management, clock synchronization and other issues. In this paper, a novel wireless sensor network synchronization sleep strategy is proposed based on the above research. This strategy uses the ZigBee-WIFI cooperative clock synchronization mechanism. The principle is that the 802.15.4 wireless sensor can detect beacon frames of WiFi broadcasts in the same frequency band and use them as the reference clock to correct the local clock. This approach has several key advantages: (1) do not need to make any changes to the 802.11 AP, so you can use the ubiquitous WiFi. (2) coverage is large. Measurements show that many WiFi AP products, even in a very complex environment, still have a communication range of around $100 \mathrm{~m}$. (3)802.15.4 wireless sensor equipment distribution than AP-intensive, so many wireless sensor network nodes can use the same beacon signal as a 
reference clock. Compared with the conventional clock synchronization algorithm, this method cannot synchronize the node time through the exchange of information. This time synchronization algorithm has a greater improvement in energy consumption and clock synchronization accuracy than other synchronization protocols.

\section{Conclusion}

In this paper, an optimized intra-group, inter-group power and slot resource allocation algorithm is proposed for cluster-based time-division cooperative MIMO communication systems in wireless sensor networks. By adjusting the time slot and power distribution parameters, the maximum data throughput is guaranteed while the reliability requirements are met. In addition, this paper establishes a variety of transmission mode energy consumption model, proposed a new path loss based on the transmission mode switching strategy. According to the geometric position of the cooperative node and the relay node in the cooperative communication, the communication system can switch between SISO, cooperative MISO, single hop, multi-hop and other transmission modes. The existence domain of the cooperative node and the relay node is also obtained by solving the conversion threshold. The size of the switching threshold is determined by the energy consumption of the sensor nodes in the system. More energy-efficient circuit modules bring smaller mode switching thresholds. In principle, any distance of the wireless sensor network communication transmission can be decomposed into the optimal single-hop or multi-hop model of the sequential combination.

\section{Acknowledgment}

This work is supported by Scientific and Technological Research Program of Chongqing Municipal Education Commission(Grant No. KJ1729404).

\section{References}

[1] Huifang Zhou: Journal of Information, Vol. 6 (2004) No 53, p.25-26

[2] Hongli Zhang: Computer Education, Vol. 12 (2005) No 27, p.74-76

[3] Qin Guo: Automation and instrumentation, Vol. 1 (2006) No 33, p.11-14

[4] Jieming Liu: Guangxi Normal University, Vol. 3 (2007) No33, p.121-124

[5] Jia Xinzhang, Li Jingyuan. Electronic Industry, Vol. 6 (2014) No 53, p.25-26 